Session 1125

\title{
Development of a Spacecraft Detail Design Course - Incorporating Design, Build, Integration and Test
}

\author{
Dr. Rachel Shinn, Dr. James Lyall \\ Embry Riddle Aeronautical University, Prescott, AZ
}

\begin{abstract}
With a few notable exceptions, spacecraft are never composed of a single subsystem. Typically, they comprise multiple subsystems that interact with each other. For example, it is very common for even small satellites to include power, communications, attitude, structure and payload subsystems, interacting to make up the entire system. The one-semester detail design course is a follow on course to a one-semester preliminary design course. Together, they constitute the program capstone design component. In detail design the principles of design are taught and applied to the multiple subsystems and integration required for spacecraft. This requires consideration of the practical requirements and constraints typically required for spacecraft. It is important to note that the design course employs the entire class as a single team, and that the final result of the course is a design and tested prototype of an integrated spacecraft model.
\end{abstract}

The objectives of the Spacecraft Detail Design course are to 1) teach the detailed design process, including analysis leading to configuration managed specification drawings, assembly drawings, requirements and test plans; 2) teach the students to integrate multiple subsystems into a system design; 3) give the students the experience of building their design as a prototype and 4) complete the design process by having the students test their prototype against the design requirements.

This paper outlines the way in which these objectives are being met and the pedagogical tools that have been developed in the process.

\section{Introduction}

Design, build, fly has been done in both the spacecraft and the aircraft arena. Typically, this is done over multiple semesters. We complete this cycle in a single semester. Some schools that have design, build, fly programs are $\mathrm{MIT}^{1}$, Purdue ${ }^{2}$, and USAFA ${ }^{3}$. At Embry-Riddle Aeronautical University, we have a two-semester design sequence with the first semester being a 
paper design of an entire spacecraft and mission. The second semester is dedicated to design, build, integration and test. In order to accomplish this in a single semester, only a few subsystems can be done in detail, so the project is de-scoped from the previous semester's design to be some portion of the design that the students can complete in a single semester.

The benefits of this design, build, integrate and test experience are many. The student is introduced to and required to use the engineering practice of submitting their design in a configuration-managed way. They have the opportunity to utilize their education in previous coursework to drive the design of their sub-systems. They learn about the reality of connecting or machining their pieces in an effective manner. They must think about and design for integration with other subsystems that their subsystem will be required to work with. They must plan for testing of their subsystem and for testing of the integrated system. They learn about and write test plans and execute those to show their design meets the requirements. They learn to write a detailed requirements document. They learn to work with a large group and how their schedule must work with the schedule of the other subsystems in the design. Most importantly, the student has the opportunity to see if all of their training in engineering that they have put to use on the design, is actually effective in producing a working prototype.

Projects that the students have done are an orbital debris detector, integrated into a powered structure; a cold gas thruster system integrated into a structure that is required to spin; a scaled down lunar lander; portions of their design of a Mars deployable greenhouse and a solar tracking satellite model. The projects are scaled down in such a way that the some aspect of the design is a "proof of concept" for some key portion of their design from the previous semester.

The following sections will discuss the implementation of the different phases and how these phases fit in the course with the associated challenges.

\section{Design Elements and Engineering Practice}

The process of design consists of designing to set requirements, supporting the design with analysis, creating test procedures that support the requirements, creating sub-assemblies and assembly procedures that make the construction of the design straight-forward, creating part drawings and procurement specifications for all of the components of the project, creating bills of material for all sub-assemblies and for the final assembly and tying this all together with a product structure tree that shows schematically how the parts are assembled. The engineering practice that is taught consists of ensuring all drawings are completely and unambiguously specified, structuring all documentation in a controlled environment where drawings are assigned a part number, submitted for review and released into the controlled system when approved.

The students are responsible for writing a requirements document that is updated and maintained throughout the semester. It starts with top-level requirements and as the design gets refined, the requirements also get more specific. They are also responsible for test plans that verify each of the requirements. Tying the test plans to the requirements document helps the students to make the requirements document specific and written in a way that the design is test-able. After the 
students finish building their project, they will test the prototype to verify that it meets the requirements stated in the requirements document.

The students are required to think through how the project will integrate. They must decide on sub-assemblies, which may consist of portions of different subsystems, and decide how those sub-assemblies will be built into the final project. The document used to illustrate this is the product structure tree. This shows, in block diagram format, which items are the sub-assemblies and which sub-assemblies come together to form higher level sub-assemblies until the entire project is assembled. An example is shown below.

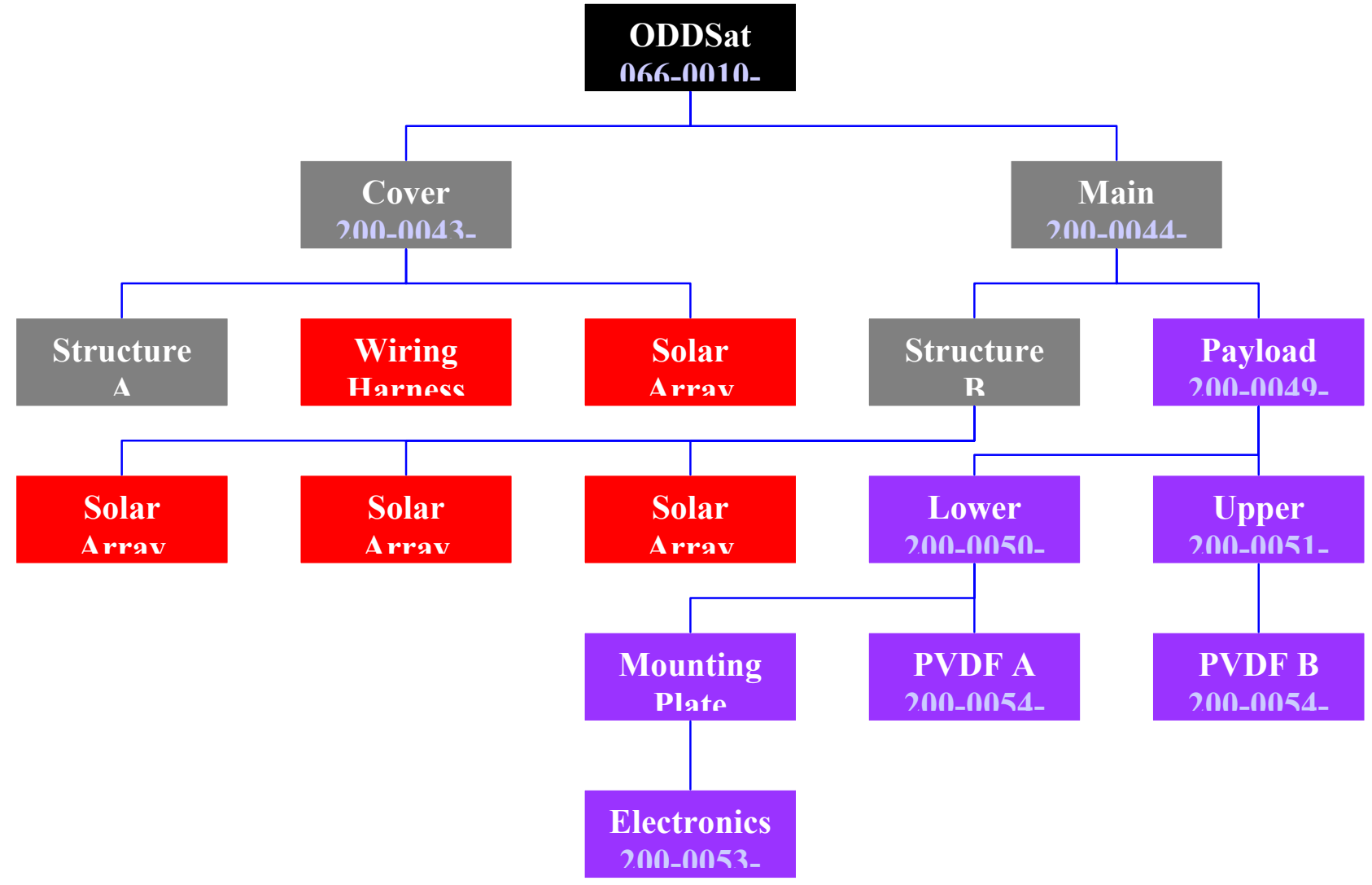

In this example, there are two main sub-assemblies that form the project called ODDSat. The "Cover" sub-assembly consists of a portion of the structure called "Structure A", a wiring harness and a solar array. The "Main" sub-assembly consists of the second portion of the structure, "Structure B" and the payload, which is the debris sensor mentioned above. Each of these are broken into further sub-assemblies. This structure helps the student conceive from the beginning of the design how all of the subsystems will be integrated into the system and how all of the parts will be assembled.

\section{Introductory Project}

In order to teach the students the design process so that they can be clear on what documentation needs to be done and what role analysis must play in their design, an introductory project was 
devised that mimics the full design process for a simple structure. The students have only two weeks to design, build and test their mini-project. Integration is negligible since there are only one or two sub-assemblies and only one subsystem.

We have tried two versions of the introductory project. The first one was for the students to design a sheet metal box with a removable lid that had a certain volume and structural requirement. This choice gave the students a requirement to design to that could be tested and the structural requirement provided the opportunity for some simple analysis to support the design.

The second project was more open. The students were required to select a portion of their structural design from the previous semester, design it in detail, design it to the maximum launch load from last semester, build it and test it to failure. The second project was an improvement over the box project, since the students are inherently more interested, and the project gets the students looking at their structural design to see how they would actually select fasteners and material to build it.

The requirements for the design are that it has at least two sub-assemblies and that it meets the maximum static launch load that was required from their previous semester's design. Once they have a design concept, they are required to size the material so that the load requirement is met with a safety factor of 2 (and no more! The tendency is to over-design). The assembly of the design must be illustrated in a product structure tree, as shown in the above example, and show all of the subassemblies and how the subassemblies integrate into the final product. Each part must be drawn using a CAD tool and the drawings inserted into a formal document template and given a part number. Assembly drawings, bills of material identifying all of the parts for a given subassembly, test plans and requirements documents are also submitted into a formal system and assigned part numbers.

When the introductory project is built, according to the design, the prototype is checked against the submitted drawings and checked for conformity. This mimics the process that will be used at the end of the semester as part of the grade for their design. After their prototype is checked against the design, the students are required to test their structure to failure and determine if it was over, under of correctly designed according to the structural requirement.

This entire process gives the students a good introduction to the design process that we expect them to follow for the main course project. The students enter the class with a good understanding of subsystems, or areas of expertise like power, thermal, structures, attitude, communications, etc, and it is often challenging to get them to switch gears and think about the details of how it will be assembled. Often, such details as how they will get to an internal part if something needs to be replaced, are not considered. This process of teaching them to think about the assembly process helps the students to see how to integrate sub-assemblies in such a way that they will be able to access the internal parts, and successfully assemble the pieces.

IV. Build, Integration and Test 
The main project follows the same process as the introductory process. The drawings must be complete before they can build, and the drawings must have been accepted into the configuration-managed system. Near the middle of the semester, their drawings should be complete and we have a review to be sure that each subsystem, subassembly and the overall project is shown to meet the given requirements and that the design is sensible and can be integrated. Faculty from the various disciplines that are involved in the project attend this review. In this way, the students are critiqued by specialists in their separate subsystems.

Once the student teams address all of the action items from the review, the build process can begin. The students use the machine shop and the machinist on campus to create their parts and assemble their subassemblies. There is subsystem testing that can begin at this stage, before the entire project is integrated. As the subassemblies get completed, the integration can begin. After the integration is complete, the final systems level testing can begin.

The structural tests consist of static loads testing, and required impact testing, shock and vibration testing. The launch vehicle environment usually drives these tests. Any additional tests depend on the given requirements and have included a center of gravity test, an inertia test, a static loads test, a spin-up test, testing of the propulsion system to its specifications, deployment tests and other functionality tests.

\section{Management Structure}

The students are broken up into subsystem teams (there are always at least two and no more than five). Each team has a representative that is also on the integration team. The integration team is responsible for ensuring that the subsystems and resultant subassemblies have appropriate interfaces and that the teams understand how they must integrate with the other subsystems and subassemblies of the project. The leader of the integration team is the project manager for the entire class. Each team has its own team leader and the team lead is responsible for ensuring that the schedule and budget are maintained and that the work is appropriately distributed on the team.

The integration team is responsible for the overall class schedule and must negotiate budget changes between teams. If one team is under budget and another, over budget, the integration team must manage the overall class budget to a fixed amount, i.e., money can be moved between teams, but the overall amount of money the class has to work with is fixed.

The integration team must also handle the class schedule. Their responsibility in this regard is to see which subassemblies must be built and tested first so that the integration can be done within schedule.

\section{Evaluation of Individuals}

Since this is a highly team based project, it is difficult, but extremely important to fairly determine individual grades. This is important from an accountability point of view as well as the obvious need to provide each individual with a grade based on his/her performance. Star 
performers on a team should get rewarded and weak performers should have their grade reflect this performance also.

The instructors evaluate the team's design and assign a team grade. Each individual evaluates their respective team members and also assigns bonus dollars to their teammates. In addition, each individual does a self-assessment ${ }^{4}$. The self-assessment gives the instructor a clear idea of the contribution of that individual and provides a possible counterpoint to their team mates' evaluations. This way, if a team member is rated poorly, but can show that they have made significant contributions to the team, the instructor can over-ride the team evaluation. Also, a team member is rated highly, but really has no contribution to merit this high rating, the instructor can modify the evaluation as well. The team evaluations are averaged for each individual and this average is compared to the overall team average. If a team member is rated above the average, their grade is increased from the team grade. If it is below, it is decreased from the team grade. This way, the individual grade can be extracted from the team grade.

The evaluation form and self-evaluation forms are shown in the following tables.

\begin{tabular}{|l|}
\hline Team Evaluation \\
\hline $\begin{array}{l}\text { Please write the names of all your team members, and rate the degree to which each member } \\
\text { fulfilled his/her team responsibilities. Do NOT rate yourself. Sign your name at the bottom. } \\
\text { The possible ratings are as follows: }\end{array}$ \\
\hline $\begin{array}{l}\text { Excellent: Consistently went above and beyond, tutored teammates, and routinely went above } \\
\text { and beyond the basic team responsibilities. }\end{array}$ \\
\hline $\begin{array}{l}\text { Very good: Consistently did what he/she was supposed to do, very well prepared and } \\
\text { cooperative. }\end{array}$ \\
\hline Satisfactory: Usually did what he/she was supposed to do, acceptably prepared and cooperative. \\
\hline \\
\hline Ordinary: Often did what he/she was supposed to do, minimally prepared and cooperative. \\
\hline \\
\hline Marginal: Sometimes failed to show up or complete assignments, rarely prepared. \\
\hline \\
\hline Deficient: Often failed to show up or complete assignments, rarely prepared. \\
\hline Unsatisfactory: Consistently failed to show up or complete assignments, unprepared. \\
\hline Superficial: Practically no participation. \\
\hline No Show: No participation at all. \\
\hline Additionally, your team has been awarded \$5000 to use as bonus money. Distribute the bonus \\
\hline
\end{tabular}




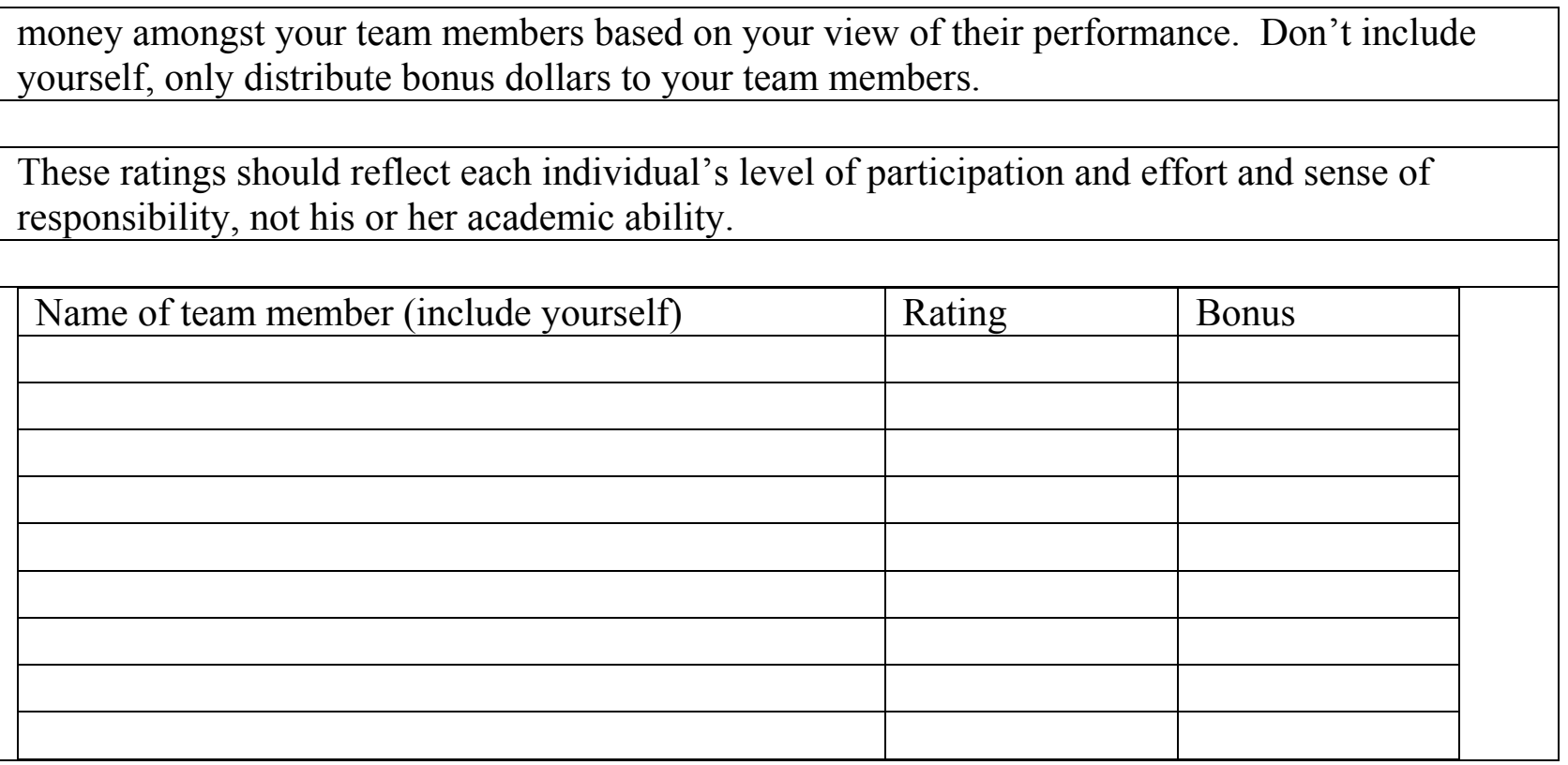

\begin{tabular}{|l|l|}
\hline \multicolumn{2}{|c|}{ Self Evaluation for Project: } \\
\hline Team Name: & Date: \\
\hline Team Member: & Principle Duty Title: \\
\hline Supervisor: & Position: \\
\hline a. State Your Significant Duties and Responsibilities: \\
\hline b. Summarize Your Major Task Completions in Fulfillment of Your Duties: \\
\hline c. List Your Significant Contributions: \\
\hline
\end{tabular}

\section{The Projects}

As mentioned earlier, projects that have been done in this course are an orbital debris detector; a cold gas thruster system integrated into a structure that is required to spin; a scaled down lunar lander; and portions of their design of a Mars deployable greenhouse.

The project in progress as of this writing is the orbital debris detector. This preliminary design consisted of an orbital debris detector, an attitude determination and control system, a power subsystem, a de-orbit system, a power system, a communications and data handling system and a structures subsystem. The detail design was de-scoped to include only the orbital debris detector, and the power and structures subsystems. The use of the introductory project, which provided the opportunity to see how analysis played into the design and how the documents 
needed to be managed, improved the overall understanding of what was required for completion of the design. With the recent acquisition of a vibrations table, the students will be able to perform modal, shock, sine and random vibrations tests on their structure.

The most complete and successful of the designs was the cold gas thruster system. The analysis, drawings, requirements document, subsystem testing, integration and system level testing were all well executed and documented. This improvement over previous years is certainly partly due to the student group that did it, but it is also due to making the system more transparent to the students and to the introductory project that they did at the beginning of the semester. The project is shown in the figure below. The cold gas thrusters were designed to be supersonic and to operate with $\mathrm{CO}_{2}$ gas; they were manufactured in our machine shop. They were able to successfully spin the spacecraft model up as required. The center of gravity and inertias were very close to the predicted values and within the ranges specified in the requirements.

The lunar lander had a deployable, inflatable antenna and could orient itself in azimuth and elevation. The spacecraft was closed up, to simulate the stowed configuration of the spacecraft in the launch vehicle, and had to autonomously open and deploy the inflatable antenna. This project turned out to be very ambitions for the time allotted for the course. The students were successful in building and testing the design, however, the documentation was not as successful. It became clear that the students needed a better introduction to the configuration management system so that their design, consisting of detailed drawings, analysis, requirements document, test plans and test reports, could be more complete.

The Mars deployable green house took the landing subsystem, the deployable, inflatable greenhouse canopy, and the ground movement subsystems from their previous semester's design for their detail design project. The landing subsystem had to autonomously deploy parachutes to slow it down, deploy airbags at the appropriate time during the descent, and separate the parachute module from the greenhouse. The greenhouse portion had to withstand the landing loads, open up and inflate the greenhouse canopy. The ground movement system was designed to inflate and deflate airbags around the greenhouse module to move it to the desired location. All of the subsystems were tested separately and successfully; and dropping the model from an airplane was the final system test. Unfortunately, a pin caught on the airplane door when it was being ejected and the parachute failed to deploy properly and the model was destroyed on landing.

\section{Summary}

The students are able to execute the entire design, build, integrate and test sequence within a single semester. Knowing what to expect with regard to the documentation and testing helps the students to select a less ambitious project and leads to a greater level of success. The projects provide the opportunity to test out concepts that are conceived of in the previous design course, resulting in a rich educational experience for the student and preparing them for the design practices they will encounter in industry. 


\section{REFERENCES:}

[1] MIT Aero/Astro Curriculum, 2004, <http://web.mit.edu/aeroastro/www/cdio/curriculum.html>

[2] Purdue University, Raisbeck Engineering, Design. Build. Test Website, 2004, $<$ http://roger.ecn.purdue.edu/ raisbeck/>

[3] United States Air Force Academy, Astronautical Engineering Program, 2004, $<$ http://www.usafa.af.mil/dfas/academics/astro_eng_program.htm>

[4] Massie, Darrell D., Massie, Cheryl A.., "Framework for Organization and Control of Capstone Design/Build Projects.," ASEE Annual Conference, Session 2559

\section{DR. RACHEL SHINN}

Dr. Rachel Shinn is an assistant professor of Aerospace Engineering at Embry-Riddle Aeronautical University in Prescott, AZ.

DR. JAMES LYALL

Dr. James Lyall is an associate professor of Electrical Engineering at Embry-Riddle Aeronautical University in Prescott, AZ. 